Archives

11 | 1993

Histoire intellectuelle // Réflexions collectives sur I'histoire sociale

\title{
Pour une nouvelle histoire sociale
}

Journées de réflexion du CRH. Paris, 14-15-16 octobre 1993

\section{Bernard Lepetit}

\section{(2) OpenEdition \\ Journals}

Édition électronique

URL : http://journals.openedition.org/ccrh/2775

DOI : $10.4000 /$ ccrh. 2775

ISSN : $1760-7906$

Éditeur

Centre de recherches historiques - EHESS

Édition imprimée

Date de publication : 10 octobre 1993

ISSN : 0990-9141

\section{Référence électronique}

Bernard Lepetit, "Pour une nouvelle histoire sociale », Les Cahiers du Centre de Recherches Historiques

[En ligne], 11 | 1993, mis en ligne le 05 mars 2009, consulté le 03 mai 2019. URL : http:// journals.openedition.org/ccrh/2775; DOI : 10.4000/ccrh.2775

Ce document a été généré automatiquement le 3 mai 2019.

Article L.111-1 du Code de la propriété intellectuelle. 


\title{
Pour une nouvelle histoire sociale
}

\author{
Journées de réflexion du CRH. Paris, 14-15-16 octobre 1993
}

\section{Bernard Lepetit}

1 Le temps parait venu d'une redéfinition (provisoire, mais non éphémère) de l'histoire sociale. La conjoncture y est propice. A l'interieur de l'histoire et parmi les disciplines voisines, des propositions émergent qui présentent les mêmes caractères : attention croissante à l'action située, aux constructions identitaires, à l'ordonnancement temporel des phénomènes. On propose donc d'explorer une nouvelle alliance qui associerait, au sein de la discipline, convention, lien social, historicité; une nouvelle hiérarchie des questions orientée par l'une d'entre elles : il s'agirait de savoir comment l'accord se fait, ou échoue à se faire.

2 Au cours de trois journées consécutives, organisée chacune autour d'un thème, un petit nombre de textes destinés à préparer la discussion seront présentés. L'approche privilégiée sera l'étude de cas: analyse critique d'un livre, compte-rendu d'une expérience de terrain individuelle ou collective, exposé de recherches récentes. Sans qu'aucun d'entre eux soit privilégié, trois axes de débat seront suivis : historiographique, épistémologique, pratique. Les débats n'auront pas simplement pour ambition de prendre acte de nouvelles configurations du savoir, mais d'en favoriser, d'une manière critique, la cristallisation en histoire. Par formation, tout historien le sait : le futur n'est pas ce qui vient vers nous, mais ce vers quoi nous allons.

\section{Normes, valeurs, conventions}

3 Normes, valeurs, conventions, constituent des représentations collectives et prennent corps dans des organisations, des institutions, des règles de droit. Le marché de l'art ou celui de la terre, les corporations de métiers ou les systèmes familiaux, les règles de dévolution de la couronne et les pratiques de la lecture s'organisent selon les règles admises d'un jeu social qu'elles donnent à voir.

4 Presque toutes, à tout moment, paraissent tirer leur force de la durée : elles apparaissent comme un cadre hérité du passé qui enserre et modèle les pratiques individuelles et 
collectives. A la limite, leur évidence partagée leur confère dans l'instant une apparente intemporalité. Pourtant, comment imaginer des normes inappliquées, des conventions qu'aucun échange ne viendrait éprouver? Normes, valeurs, conventions sont aussi le produit transitoire d'un système d'interactions individuelles : elles façonnent les accords locaux, mais se trouvent en retour façonnées par eux. Elles sont à tout moment ce que les hommes en font. Mieux : la variété des valeurs, des normes et des conventions crée un univers complexe et offre aux hommes (inégalement selon leur position et selon la conjoncture) un espace de ressources pluriel. Elle donne aux sociétés un jeu qui leur assure d'évoluer dans des directions pas nécessairement prévisibles.

5 Une première série de questions devrait porter sur la manière dont les normes se construisent et se défont, sur les tensions temporelles sur lesquelles elles s'organisent, sur leur mode d'être dans l'histoire.

6 Leur intérêt épistémologique est de ruiner deux types de distinctions réductrices : entre les faits et les représentations d'un côté ; entre la construction du fait historique et les procedures d'analyse de l'autre. Normes et conventions ont en effet un double aspect. Pour l'historien, elles sont d'une part une façon de concevoir ou de construire le réel. La fixation du salaire au xvIII siècle, par exemple, s'organise autour d'un ensemble de normes qui définissent la relation salariale spécifique du moment. Elles sont, d'autre part, une manière d'élaborer un outil de connaissance historique. Ainsi, Simmel constitue l'argent comme norme afin de donner à lire, a travers elle, une configuration du champ social.

7 Une question générale résulte de cette dualité: celle de l'interaction profonde et constitutive de savoir entre l'élaboration d'une procédure et la pensée du réel. Elle peut être spécifiée de plusieurs manières : comment attribuer le qualificatif de norme ou de convention et sur quelle base le refuser? Comment valider une démonstration quand s'estompe la distinction entre le terrain d'expérience et les hypothèses? La méthode ne conduit-elle pas à élaborer des objets à chaque fois particuliers et à devoir, pour généraliser, construire un nouvel objet de dimension et de configuration originales?

\section{Liens sociaux, identités sociales.}

$8 \mathrm{Au}$ lieu de considérer pour donnés les groupes et pour acquise l'appartenance des individus à ces groupes (ordres, classes, cités, tribus...) qui les enferment et les définissent, les sciences sociales inversent maintenant la perspective et considèrent comme problématiques les regroupements et les identifications.

9 Le renversement incite d'abord à comprendre pourquoi les anciens types d'analyse ont éprouvé si radicalement leurs limites que les approches de substitution se veulent opposées. Un groupe n'est plus composé par un ensemble d'individus caractérisés par la même série de critères (le type d'activité, le niveau de fortune, celui de l'instruction...) qui définissent une position semblable dans le champ social. Les relations multiples (politiques, économiques, sociales, culturelles...) nouées par les acteurs, les pratiques communes qu'ils développent créent des solidarités, dessinent des réseaux, définissent des identités temporaires, proposent du champ social des découpages exclusifs. L'image de la société française du xix ${ }^{e}$ siècle change de nature dès lors que l'on considère moins les positions économiques que les pratiques de l'inter-mariage. L'existence du monde 
ouvrier devient problématique, même à l'échelle d'une ville ou d'une entreprise, si l'on prend en compte la variété des parcours individuels.

10 A prendre le parti des phénomènes, on s'interrogera sur les processus de fixation des identités et d'affirmation des solidarités que des pratiques partagées ou des relations établies induisent. Ou bien l'on considérera les modalités selon lesquelles la conjoncture ou l'événement (et plus généralement l'historicité des situations) doivent être introduits dans la formation d'expériences partagées et la reconnaissance d'identités communes.

11 Mais il est particulièrement désastreux, dans ces domaines, de croire qu'on peut adopter seulement le parti des choses. La définition des identités est tout entière sociale. Elle porte non seulement la marque d'expériences individuelles et collectives, mais tout autant celle des catégories analytiques et des techniques d'observation qui les donnent à lire. L'expérience partagée du sous-emploi ne suffit pas à l'invention de la figure du chômeur. Toute procédure d'identification des relations individuelles ou des pratiques communes est productrice d'un découpage du social. Les mécanismes à travers lesquels l'historien construit des tableaux alternatifs de la société (choix de l'échelle, définition des individus, sélection des outils statistiques, si l'on se place dans le cadre de la statistique sociale) mériteraient d'être analysés finement. Ils forment une rhétorique dont il convient d'apprécier les effets.

\section{Historicité, processus, temporalité}

La prise en compte du temps fonde la singularité du travail de l'historien. Elle connaît depuis peu des bouleversements susceptibles, si l'on y prête attention, de modifier l'analyse historique dans les années à venir.

D'une part, les disciplines voisines évoluent. Usant d'échelles chronologiques différentes, l'anthropologie, la sociologie, la géographie, l'économie, connaissent un «tournant historique " : elles prêtent une plus grande attention aux conséquences de l'inscription dans la durée des phénomènes qu'elles donnent à voir. Qu'il s'agisse, par exemple, du temps long des processus identitaires, des conventions partagées et des apprentissages collectifs, ou du temps court des situations d'accord ou de conflit locaux, des modèles sociologiques nouveaux intègrent l'hypothèse que toute situation sociale est dépendante de son cheminement temporel antérieur.

Chacun des soulignements chronologiques que ces modèles opèrent, tant au niveau microanalytique des acteurs (leur compétence temporelle) qu'au niveau macroanalytique des processus (les modalités temporelles des effets de composition des phénomènes) a son prix. La graduation de l'échelle temporelle récemment mise en oeuvre par l'économie des conventions, par exemple, reste sommaire: entre la très longue durée des conventions et la succession des instants de leur mise a l'épreuve, il semble y avoir pour l'heure peu de place pour une réflexion intermédiaire. L'une des tâches des historiens pourrait consister en particulier à s'interroger sur les effets du type d'histoire et des formes de la graduation des modèles temporels mis en oeuvre par les sciences sociales. Il convient encore de déterminer comment ces temporalités, souvent associées à des concepts ou à des savoirs théoriques, peuvent rencontrer le temps de l'histoire générale pour des usages et à des fins d'abord empiriques.

Par ailleurs, le temps descriptif est progressivement mis à mal par les nouvelles méthodes statistiques. Elles déstructurent les emboîtements et les décompositions habituelles des 
séries chronologiques (le couple structure-conjoncture, ou les trois temps de la durée braudélienne) au bénéfice d'une cohérence entre les intentions de l'observateur, les choix dans l'usage de la méthode et le réel quantifié. L'historien se trouve ainsi confronté à une validation circulaire du savoir qui oblige à réexaminer, à nouveaux frais, les règles de création de la connaissance historique.

Ces trois questionnaires se renvoient les uns aux autres, se recoupent partiellement. Il faut y voir un avantage. De l'unité du questionnement, on attendra la capacité de l'entreprise à jouer un rôle d'ordonnancement dans la pratique diverse, et riche, du métier d'historien.

\section{PROGRAMME}

18 Jeudi 14 octobre 1993 - normes, valeurs, conventions Matin : $9 \mathrm{~h}-12 \mathrm{~h}$. Présidence : Lucette VALENSI Roger CHARTIER, HISTOIRE DES SCIENCES ET HISTOIRE CULTURELLE : UNE NOUVELLE RENCONTRE ?

Jocelyne DAKHLIA, DISCRIMINANTS CULTURELS ET MODÈLES DE SOUVERAINETÉ.

Après-midi : $14 \mathrm{~h}-17 \mathrm{~h}$. Présidence : Jean ANDREAU

Simona CERUTTI, QUELQUES RÉFLEXIONS AUTOUR DU RAPPORT ENTRE PRATIQUES ET NORMES.

Alain DEWERPE, LA RÈGLE ET LE STANDARD : CONVENTION DU PRODUIT ET CONVENTION DE

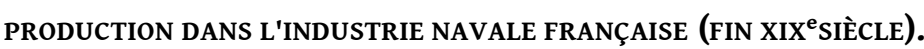

Vendredi 15 octobre 1993 - Liens sociaux, identités sociales

Matin : $9 \mathrm{~h}-12 \mathrm{~h}$. Présidence : Patrice BOURDELAIS

Nancy GREEN, CLASSES ET ETHNICITÉ : DES CATÉGORIES CADUQUES DE L'HISTOIRE SOCIALE ?

Maurizio GRIBAUDI, APPROCHE CONFIGURATIONNELLE ET ANALYSE DU SOCIAL.

Après-midi : $14 \mathrm{~h}-17 \mathrm{~h}$. Présidence : Arlette FARGE

Christiane KLAPISCH, ENTRE OSTRACISME ET ASSIMILATION : LES INDICATEURS SOCIAUX D'UN GROUPE MEDIEVAL TEL QUE LES HISTORIENS, LES CONTEMPORAINS ET EUX-MÊMES L'ONT DÉFINI. Jacques REVEL, L'INSTITUTION ET LE SOCIAL.

Samedi 16 octobre 1993 - Historicité, processus, temporalités

Matin : 9 h-12 h. Présidence : Maurice AYMARD

Alain BOURREAU, COMMENT ORDONNER LES MODALITÉS TEMPORELLES DES REPRÉSENTATIONS.

Jean-Yves GRENIER, CONSTRUIRE ET DÉCONSTRUIRE LES TEMPORALITÉS EN HISTOIRE.

Après-midi : $14 \mathrm{~h}-17 \mathrm{~h}$. Présidence : Jean-Claude PERROT

André BURGUIERE, DE LA NOTION DE CHANGEMENT ET DE SES RAPPORTS À LA TEMPORALITÉ.

Bernard LEPETIT, LE PRÉSENT DE L'HISTOIRE. 